\title{
Model independence of scattering of three identical bosons in two dimensions
}

\author{
Sadhan K. Adhikari \\ Instituto de Física Teórica, Universidade Estadual Paulista, 01405 São Paulo, São Paulo, Brazil \\ A. Delfino \\ Department of Physics and Astronomy, University of Maryland, College Park, Maryland 20742 \\ and Departamento de Física, Universidade Federal Fluminense, Niterói, Rio de Janeiro, Brazil \\ T. Frederico \\ Instituto de Estudos Avançados, Centro Técnico Aeroespacial, 12225 São José dos Campos, São Paulo, Brazil \\ Lauro Tomio \\ Instituto de Física Teórica, Universidade Estadual Paulista, 01405 São Paulo, São Paulo, Brazil
}

(Received 4 May 1992)

\begin{abstract}
Within the framework of scattering integral equations in momentum space, we present numerical results of scattering of three identical bosons at low energies in two dimensions for short-range separable potentials. An analysis of the present numerical results reveals the three-particle scattering observables to be independent of potential shape provided the low-energy two-particle binding energy and scattering length are held fixed throughout the investigation. We think that the present conclusion of model independence will be valid for any potential, local or nonlocal, whose range is much smaller than the size of the two-particle bound state.
\end{abstract}

PACS number(s): 03.65.Nk

\section{INTRODUCTION}

Over the past decade there has been a great deal of activity in studying the three-particle problem in two space dimensions both theoretically [1-6] and experimentally [7-10] due to a variety of reasons. Experimentally, there has been considerable interest in two-dimensional systems such as helium adsorbed on graphite [10] and spinpolarized hydrogen $(\mathrm{H} \downarrow)$ [5,7-9] recombining on a helium film. Also, in view of the possibility of detecting multiparticle bound states on monolayers of quantum gases [10], it is interesting to study numerically the quantummechanical few-particle bound-state problem.

In the recent past there has been a significant amount of theoretical studies [11] in the quantum and statistical mechanics of anyons, which are particles having continuous fractional spin and thus interpolating and simulating between boson and fermion properties. Anyons exist only in two dimensions and this has increased the relevance of few-particle problems in two dimensions. As the anyons represent bosons in one of the extreme limits a study of the quantum-mechanical three-boson system is likely to enhance the understanding of anyon properties.

A knowledge of the complete spectrum of the Hamiltonian for the few-particle systems in two dimensions is essential for studying the quantum-cluster coefficients in two dimensions [12]. For a confining potential the complete spectrum constitutes of bound states only, whereas in the nonconfining case one has to deal with both bound states and scattering. Such studies have been undertaken $[11,12]$ for bosons, fermions, and anyons in general and also in the limits of high and low temperatures. A knowledge of the complete spectrum of the $n$-particle Hamiltonian is necessary for the study of the $n$th quantum-cluster coefficient [12]. At the low- (high-)temperature limit one needs such a knowledge at low (high) energies.

Hence, a systematic study of the quantum-mechanical few-particle system in two space dimensions will shed light on all the above-mentioned problems. Here we report numerical results on both scattering and boundstate observables at low energies for the quantummechanical three-boson system with a special objective in mind. We would like to investigate how sensitive are these observables to variations of the interaction potential while maintaining two-particle binding energy and scattering length fixed under certain restrictions. The restrictions are those of short-range potentials where the typical few-particle bound-state size is much larger than the range of the interaction potential. We would like to study the few-particle system under such restriction partly because of previous studies indicating that this is the situation in many atomic and chemical systems $[1,2]$, and partly because of the simplicity in the treatment of the few-particle problem in two dimensions via the momentum-space connected kernel integral equations using such short-range potentials. Also, under these restrictions and at low energies the result of such a study is expected to be universal and independent of the details of the potential model employed [13].

There have been certain calculations [1-6] for fewparticle bound states in two dimensions. Bruch and Tjon $[1,2]$ were the first to investigate the model dependence 
of the three- and four-particle bound states on the shape of the interaction potentials. They compared the results of separable potential calculations with those employing the Lennard-Jones potential. They concluded that the results were sensitive to the potential model employed as they varied the strength of the potential. Later on in relation to the study of Efimov and Thomas [14] effects in two dimensions Adhikari et al. [4] pointed out that the study of Bruch and Tjon is not entirely to the point and one should maintain the two-particle low-energy observables - both binding energy and scattering length - fixed in studying the above model dependence. Once thi is done the above model dependence of few-particle bound-state observables in two dimensions reduces significantly. This has been related to the absence [4] of Efimov and Thomas effects in two dimensions.

In this work we complement the study of Ref. [4] in two dimensions on the model independence of three-particle bound states on potential models and present a systematic study of the low-energy scattering of a boson on the bound state of two identical bosons. We consider three identical bosons interacting via pairwise $S$-wave separable potentials with simple form factors. We calculate the $S$-wave scattering length and phase shifts for the scattering of a boson on the bound state of two others. We investigate the sensitivity of our result on variation of the shape of interaction potential. Presently, nothing is known about the scattering observables of three particles in two dimensions. Our study will provide some idea of the scattering in the three-boson system.

Though we have limited the present study to a class of separable potentials, we think that the present conclusion of model independence of scattering of three identical bosons in two dimensions will have a much wider range of validity provided that the range of the twoparticle potential is much smaller than the size of the two-particle bound state. The present conclusion is similar to the existence of the well-known effective-range expansion for the low-energy two-particle problem interacting via short-range potentials. The result of low-energy two-particle scattering in three dimensions is essentially determined by two parameters and is independent of the shape of the potential - local or nonlocal. A similar model independence is observed in the low-energy threeparticle system in three dimensions [4], where the result is determined by three parameters - two-particle binding energy and scattering length and three-particle binding energy - and is independent of the potential shape. We find in the present work that the low-energy threeparticle scattering in two space dimensions is essentially determined by two parameters and is independent of the shape of the potential. This is what we call the model independence in the present context. Deviation from this model independence has occurred in our study, as we shall see in Sec. III, when we are away from the shortrange potential limit. Use of realistic potentials is not expected to change the present conclusion provided that the potentials are of the short-range type. Bruch and Tjon [1], in their study of the three-boson bound-state problem in two dimensions, observed that the results of Lennard-Jones and separable potentials have the same trend.

Through our numerical study we establish that the three-particle bound state and scattering observables at low energies in two dimensions are quite insensitive to variations of the interaction potential provided that the two-particle binding energy and the scattering length are held fixed. This is not quite so in three dimensions where the low-energy scattering and bound-state observables for three- and few-particle systems are sensitive to the details of the potential models employed or to the shape of the interaction potentials even if the low-energy twoparticle observables are held fixed.

We have confined the present study to the $S$-wave identical bosons but our conclusions are expected to hold true in the case of fermionic systems and higher partial waves also. This is because few-fermion systems obey scattering integral equations quite similar to the few-boson systems with a somewhat weakened effective interaction as a consequence of the Pauli principle between identical fermions. Because of the Pauli repulsion between identical fermions the few-fermion system is expected to be less sensitive to the interaction potential than the fewboson system and our conclusions should extrapolate to the fermionic case. Also, the higher partial waves of the three-particle system are expected to be weakly attractive because of the centrifugal barrier, and so are expected to be insensitive to the two-particle potential.

The present result should have interesting consequences on the calculation of quantum-cluster coefficients, surface recombination rates of spin-polarized hydrogen atoms, and the formation of helium clusters in two dimensions. Our calculations and the general arguments of Ref. [4] indicate that the results of such calculations should be independent of potential shape provided that the low-energy two-particle observables are held fixed.

In Ref. [4] the model (in)dependence of few-particle observables in different space dimensions has been related to the divergence of the trace of the momentumspace kernel of the scattering integral equation. This divergence is reduced as one moves from higher to lower dimensions as a result of the reduction of the momentumspace phase space $-d^{n} k$ for $n$ dimensions. In one dimension the bound state and scattering results are expected to be even more insensitive to potential shape. The calculation of Dodd [15] indicates this for three-particle bound states in one dimension.

In Sec. II we present the two-particle separable potential model which we employ for the calculation of the three-particle system and also some results for twoparticle scattering. Then we develop the three-particle dynamical equations which we use for the numerical study of three-particle scattering in two dimensions. An account of the numerical method and a suitable definition of the scattering phase shifts are also given in this case. In Sec. III we present and discuss our numerical results. In Sec. IV we present a summary and concluding remarks.

\section{THE MODEL}

Let us consider three identical bosons in twodimensional space interacting via the following $S$-wave 
separable potential in momentum space:

$$
\langle\mathbf{p}|V| \mathbf{k}\rangle=-\lambda g(p) g(k),
$$

with

$$
g(p)=\left(\beta^{2}+p^{2}\right)^{-m} .
$$

One of our main interests in this work is to study the sensitivity of few-particle observables to variations of potential shape, and this will be achieved by varying $m$ from 1 to 10 . For potential (1) the scattering $t$ matrix is given by

$$
\langle\mathbf{p}|t(s)| \mathbf{k}\rangle=\tau(s) g(p) g(k),
$$

with

$$
\tau(s)=-\left[\frac{1}{\lambda}+2 \pi \int_{0}^{\infty} \frac{q d q g^{2}(q)}{k^{2}-q^{2}+i 0}\right]^{-1},
$$

where $s=\hbar^{2} k^{2} / 2 \mu, \mu$ is the reduced mass of the twoboson system, $s$ is the center-of-mass energy, and $k$ is the on-the-energy-shell (on-shell) relative wave number. In this work we shall employ units $\hbar=2 \mu=1$. The fully on-shell $t$ matrix $\left\langle k\left|t\left(k^{2}\right)\right| k\right\rangle=2 \pi\left\langle\mathbf{k}\left|t\left(k^{2}\right)\right| \mathbf{k}\right\rangle, k^{2}=s$, is given by

$$
\begin{aligned}
\left\langle k\left|t\left(k^{2}\right)\right| k\right\rangle= & {\left[-\frac{\left(\beta^{2}+k^{2}\right)^{2 m}}{2 \pi \lambda}+\ln \left(\frac{\beta}{k}\right)\right.} \\
& \left.-\sum_{j=1}^{2 m-1} \frac{\left(\beta^{2}+k^{2}\right)^{j}}{2 j \beta^{2 j}}+\frac{i \pi}{2}\right]^{-1} .
\end{aligned}
$$

The two-particle bound states at negative energies are given by the vanishing of the quantity in the square bracket. Using the effective-range expansion, this $t$ matrix is usually parametrized by [16]

$$
\left\langle k\left|t\left(k^{2}\right)\right| k\right\rangle=\frac{2}{\pi\left(-\cot \delta_{2}+i\right)}
$$

with

$$
\cot \delta_{2}=a_{2}+(1 / \pi) \ln \left(k^{2}\right)+b k^{2}+c k^{4}+\cdots .
$$

Here $\delta_{2}$ is the scattering phase shift and $a_{2}$ is the twoparticle scattering length. There is some ambiguity in defining $a_{2}$ in two dimensions because of the $\ln \left(k^{2}\right)$ term; the numerical value of $a_{2}$ depends on the scale used to measure the energy $k^{2}$. In our study we shall be measuring energy in units of the two-particle binding energy $B_{2}$ and the scattering length $\bar{a}_{2}$, defined by

$$
\cot \delta_{2}=\bar{a}_{2}+\frac{1}{\pi} \ln \frac{k^{2}}{B_{2}}+b^{\prime} \frac{k^{2}}{B_{2}}+\cdots,
$$

is related to that of Eq. (7) by

$$
\bar{a}_{2}=a_{2}+\frac{1}{\pi} \ln B_{2} .
$$

The scattering length $\bar{a}_{2}$ will be useful in our study of model independence of a three-particle bound state and scattering observables in two dimensions. When the twoparticle binding energy $B_{2}=1, \bar{a}_{2}$ reduces to $a_{2}$. In the low-energy limit, for the $t$ matrix of Eq. (5), $\cot \delta_{2}$ is given by

$$
\cot \delta_{2}=\frac{\beta^{4 m}}{\pi^{2} \lambda}+\frac{1}{\pi} \ln \frac{k^{2}}{\beta^{2}}+\frac{1}{\pi} \sum_{j=1}^{2 m-1} \frac{1}{j},
$$

so that the scattering length $\bar{a}_{2}$ is written as

$$
\bar{a}_{2}=\frac{\beta^{4 m}}{\pi^{2} \lambda}+\frac{1}{\pi} \ln \frac{B_{2}}{\beta^{2}}+\frac{1}{\pi} \sum_{j=1}^{2 m-1} \frac{1}{j} .
$$

With this discussion of the two-particle system we now present a discussion of the three-boson Faddeev equations $[17,18]$ in two dimensions for separable potentials $(1)$. In this case the Amado-Lovelace-Mitra equation [18], which is the special case of Faddeev equations for separable potentials, is given by

$$
\begin{aligned}
\langle\mathbf{q}|T(s)| \mathbf{k}\rangle= & \langle\mathbf{q}|V(s)| \mathbf{k}\rangle \\
& +\int d \mathbf{p}\langle\mathbf{q}|V(s)| \mathbf{p}\rangle \tau\left(s-3 p^{2} / 4\right)\langle\mathbf{p}|T(s)| \mathbf{k}\rangle,
\end{aligned}
$$

where

$$
\langle\mathbf{q}|V(s)| \mathbf{p}\rangle=2 \frac{g(|\mathbf{q}+\mathbf{p} / 2|) g(|\mathbf{p}+\mathbf{q} / 2|)}{s-q^{2}-p^{2}-\mathbf{p} \cdot \mathbf{q}+i 0} .
$$

Here $T(s)$ is the $t$ matrix for the scattering of a boson on the bound state of two others at a three-particle center of mass energy $s$, and $V(s)$ is the energy-dependent effective potential in this case. The on-shell wave number $k$ in this case is given by

$$
s=3 k^{2} / 4-B_{2},
$$

where $3 k^{2} / 4$ is the relative kinetic energy.

The partial $S$-wave projection of the Amado-LovelaceMitra equation (12) is written as

$$
\begin{aligned}
\langle q|T(s)| k\rangle= & \langle q|V(s)| k\rangle \\
& +\int_{0}^{\infty} p d p\langle q|V(s)| p\rangle \tau\left(s-3 p^{2} / 4\right)\langle p|T(s)| k\rangle,
\end{aligned}
$$

where

$$
\langle q|V(s)| p\rangle=\int_{0}^{2 \pi} d \theta\langle\mathbf{q}|V(s)| \mathbf{p}\rangle .
$$

The equation for the partial-wave $t$ matrix (15) above has a singularity in the kernel in the $\tau\left(s-3 p^{2} / 4\right)$ term whenever $p$ equals the on-shell wave number $k$. In order to make this singularity more explicit we introduce a modified $t$ matrix and interaction potential in the AmadoLovelace-Mitra equation via

$\langle q|\bar{T}(s)| p\rangle=-\left(k^{2}-q^{2}\right) \tau\left(s-3 q^{2} / 4\right)\langle q|T(s)| p\rangle$,

and 
$\langle q|\bar{V}(s)| p\rangle=-\left(k^{2}-q^{2}\right) \tau\left(s-3 q^{2} / 4\right)\langle q|V(s)| p\rangle$.

The condition for the two-particle system to have a bound state of binding energy $B_{2}$ is given by

$$
\frac{1}{\lambda}=2 \pi \int_{0}^{\infty} p d p \frac{g^{2}(p)}{B_{2}+p^{2}}
$$

With this condition the expression (17) for $\bar{T}(s)$ above is rewritten as

$$
\langle q|\bar{T}(s)| p\rangle=\left[\frac{3 \pi}{2} \int_{0}^{\infty} p^{\prime} d p^{\prime} \frac{g^{2}\left(p^{\prime}\right)}{\left(B_{2}+{p^{\prime}}^{2}\right)\left(s-3 q^{2} / 4-{p^{\prime}}^{2}+i 0\right)}\right]^{-1}\langle q|T(s)| p\rangle
$$

We have a similar equation for $\bar{V}(s)$.

In terms of these modified potentials and $t$ matrix the Amado-Lovelace-Mitra equation becomes

$$
\langle q|\bar{T}(s)| k\rangle=\langle q|\bar{V}(s)| k\rangle+\int_{0}^{\infty} p d p \frac{\langle q|\bar{V}(s)| p\rangle\langle p|\bar{T}(s)| k\rangle}{\left(p^{2}-k^{2}-i 0\right)} .
$$

For energies below the three-particle breakup threshold the potential $\bar{V}(s)$ is real and the analytic structure of Eq. (21) is identical to that of the two-particle LippmannSchwinger equation. We employ a subtraction in the kernel of this equation in order to eliminate the singularity of the kernel. The nonsingular equation we consider for an auxiliary matrix $\Gamma(s)$ can be written as [17]

$$
\begin{aligned}
\langle q|\Gamma(s)| k\rangle= & \langle q|\bar{V}(s)| k\rangle \\
& +\int_{0}^{\infty} p d p \frac{\langle q|\bar{V}(s)| p\rangle-\langle q|\bar{V}(s)| k\rangle}{\left(p^{2}-k^{2}\right)} \\
& \times\langle p|\Gamma(s)| k\rangle .
\end{aligned}
$$

In Eq. (22) we have performed a specific subtraction in the kernel. This specific subtraction is a special case of a more general subtraction scheme for calculating the fully off-shell scattering $t$ matrix in terms of the solution of an auxiliary nonsingular integral equation [17]. As the integral in Eq. (22) is nonsingular the $i 0$ prescription in the denominator has been dropped. In the present study we shall be only concerned with the on-shell $t$ matrix which is expressed in terms of the auxiliary matrix $\Gamma(s)$ via [17]

$$
\langle k|\bar{T}(s)| k\rangle=\frac{\langle k|\Gamma(s)| k\rangle}{1-\int_{0}^{\infty} q d q \frac{\langle q|\Gamma(s)| k\rangle}{\left(q^{2}-k^{2}-i 0\right)}},
$$

which can be rewritten as

$$
\begin{aligned}
& \langle k|\bar{T}(s)| k\rangle \\
& \quad=\frac{\langle k|\Gamma(s)| k\rangle}{1-\int_{0}^{\infty} d q \frac{q\langle q|\Gamma(s)| k\rangle-k\langle k|\Gamma(s)| k\rangle}{\left(q^{2}-k^{2}\right)}-\frac{i \pi\langle k|\Gamma(s)| k\rangle}{2}} .
\end{aligned}
$$

In arriving at Eq. (24) we have separated the principalvalue and the imaginary parts of Eq. (23) and have added a term to the principal-value part which is zero. The re- sultant integral of Eq. (24) is not singular and hence does not need the principal-value prescription for its evaluation.

Comparing with Eq. (6) we identify $\langle k|\bar{T}(s)| k\rangle$ as the physical $t$ matrix for the scattering of one boson on the bound state of two others and the phase shift for this scattering, $\delta_{3}$, is given by

$$
\begin{aligned}
\cot \delta_{3}= & \frac{2}{\pi\langle k|\Gamma(s)| k\rangle} \\
& \times\left[1-\int_{0}^{\infty} d q \frac{q\langle q|\Gamma(s)| k\rangle-k\langle k|\Gamma(s)| k\rangle}{\left(q^{2}-k^{2}\right)}\right] .
\end{aligned}
$$

This expression for $\cot \delta_{3}$ should diverge as $(1 / \pi) \ln \left(3 k^{2} / 4\right)$ for small $k$. In order to calculate the scattering length $a_{3}$ for the scattering of one boson on the bound state of two others we have to extract this divergent part of $\cot \delta_{3}$ and take the limit $k \rightarrow 0$. The integral in Eq. (25) is identically rewritten as

$$
\begin{aligned}
& \int_{0}^{\infty} d q \frac{q\langle q|\Gamma(s)| k\rangle-k\langle k|\Gamma(s)| k\rangle}{\left(q^{2}-k^{2}\right)} \\
& \quad=\int_{0}^{\infty} d q \frac{\langle q|\Gamma(s)| k\rangle}{q+k}+k \int_{0}^{\infty} d q \frac{\langle q|\Gamma(s)| k\rangle-\langle k|\Gamma(s)| k\rangle}{q^{2}-k^{2}}
\end{aligned}
$$

It is easy to see that the first term on the right-hand side of this equation gives the $\ln k$ divergence for small $k$ and the second term vanishes in this limit. In order to extract the divergent term for small $k$ in expression (26) we rewrite the first term on the right-hand side, for example, as

$$
\begin{aligned}
\int_{0}^{\infty} & d q \frac{\langle q|\Gamma(s)| k\rangle}{(q+k)} \\
= & \int_{0}^{\infty} d q \frac{\langle q|\Gamma(s)| k\rangle(1+q)-\langle 0|\Gamma(s)| k\rangle}{(1+q)(q+k)} \\
& +\langle 0|\Gamma(s)| k\rangle \int_{0}^{\infty} \frac{d q}{(1+q)(q+k)}
\end{aligned}
$$

Equation (27) is just one way of separating the divergent part from the finite part of the first integral on the righthand side of Eq. (26) in the limit $k \rightarrow 0$. The first 
term on the right-hand side of Eq. (27) is finite in this limit whereas the last term, which can now be evaluated analytically, diverges in this limit. This last term of Eq. (27) is immediately evaluated to yield $\langle 0|\Gamma(s)| k\rangle \int_{0}^{\infty} \frac{d q}{(1+q)(q+k)}=\frac{\langle 0|\Gamma(s)| k\rangle}{k-1} \ln k$.

Now the expression (25) for $\cot \delta_{3}$ is rewritten as

$$
\cot \delta_{3}=\frac{2}{\pi\langle k|\Gamma(s)| k\rangle}\left[1-\int_{0}^{\infty} d q \frac{\langle q|\Gamma(s)| k\rangle(1+q)-\langle 0|\Gamma(s)| k\rangle}{(1+q)(q+k)}-k \int_{0}^{\infty} d q \frac{\langle q|\Gamma(s)| k\rangle-\langle k|\Gamma(s)| k\rangle}{q^{2}-k^{2}}-\frac{\langle 0|\Gamma(s)| k\rangle}{k-1} \ln k\right]
$$

The analog of the effective-range expansion (7) in this case is given by

$$
\cot \delta_{3}=a_{3}+(1 / \pi) \ln \left(3 k^{2} / 4\right)+b\left(3 k^{2} / 4\right)+c\left(3 k^{2} / 4\right)^{2}+\cdots,
$$

with $3 k^{2} / 4$ playing the role of the relative kinetic energy, which in the two-particle case given by Eq. (7) is $k^{2}$.

The scattering length for scattering of one boson on the bound state of two others, $a_{3}$, is now defined as

$$
a_{3}=\frac{2}{\pi\langle 0|\Gamma(0)| 0\rangle}\left[1-\int_{0}^{\infty} d q \frac{(1+q)\langle q|\Gamma(0)| 0\rangle-\langle 0|\Gamma(0)| 0\rangle}{q(1+q)}-\frac{\langle 0|\Gamma(0)| 0\rangle}{2} \ln (3 / 4)\right] .
$$

We can redefine $a_{3}$ in units such that $B_{2}=1$ as in Eq. (9),

$$
\bar{a}_{3}=a_{3}+\frac{\ln \left(B_{2}\right)}{\pi} .
$$

In Eq. (32) $\bar{a}_{3}$ so defined is the scattering length for $B_{2}=1$.

\section{RESULTS AND DISCUSSIONS}

In this section we present numerical results at low energies below the three-particle breakup threshold for scattering and bound states involving three identical bosons in two dimensions. One of the purposes of this study is to investigate the sensitivity of these results for scattering and bound states on the shape of the two-particle interaction potential while the low-energy two-particle observables, the binding energy $B_{2}$, and the scattering length $a_{2}$, are maintained constant. In some areas, such as nuclear physics, such a variation of the potential is called an "off-shell" variation, as the potential is varied in such a way that the (low-energy) two-particle on-shell observables are maintained constant. Usually, the features of the potential well known from physical considerations, e.g., the range, is held fixed in such a study while the shape is varied. We simulate such a variation by varying the constant $m(=1,2,4$, and 10$)$ of the potential form factor (2).

Because of considerations of Ref. [4] the results of three-boson bound-state and scattering calculations at low energies should depend only on the two-boson observables $B_{2}$ and $a_{2}$ for $B_{2}^{1 / 2} \ll \beta$, where $\beta$ is the range parameter introduced in Eq. (2). Under this condition the behavior of the three-particle system is expected to be universal, independent of the detail of the interaction potential. Due to the low binding of the two-particle system, the three particles are expected to spend most of the time outside the range of the potential. This model independence of the three-particle system increases as the dimension of the space is reduced. In three-dimensional space there is a reasonably strong model dependence, whereas in two-dimensional space this model dependence is non-existent at least at low energies and in the weakbinding limit [4].

Another way to understand this model independence of three-particle observables in two dimensions is achieved through a consideration of the dynamical equations (15). In the three-dimensional equivalent of Eq. (15) the convergence at the upper limit of the integral in this equation is achieved by the potential form factors $g$ 's through Eq. (13). The integral in Eq. (15) diverges as the form factors $g$ 's are set equal to unity, which corresponds to the two-particle zero-range interaction; this divergence has been related to the appearance of the Thomas effect in the case of the zero-range potential [4]. Consequently, in three dimensions the solution of Eq. (15) depends on the shape of the potential form factors. The integral in Eq. (15) in two space dimensions, because of the reduced phase space, converges even when the potential form factors $g$ 's are set equal to unity $[1,4]$. The solution of Eq. (15) in this case is expected to be insensitive, or at best very weakly sensitive, to the shape of the potential form factors. It is important to note that in the limit $\beta \rightarrow \infty$ (the potential range going to zero) Eq. (15) produces a convergent result for three particles in two dimensions. Consequently, the Thomas effect is absent, which implies that the short-range or the large momentum parts of Eq. (15), expected to be the region most sensitive to the offshell variation of the potential, have no significant relevance on the three-particle low-energy observables in two dimensions.

In our calculations we utilize $\beta=1$ and vary $\lambda$, so that $B_{2} \ll 1$, which is expected to be the domain of model independence discussed above. We vary the two-particle scattering length $a_{2}$ and the two-particle binding energy $B_{2}$ during the actual calculation. However, we would like 
to have results for fixed $a_{2}$ and $B_{2}$ in order to study the model independence of three-particle observables. This is achieved in a simple fashion. The three-boson results for $\beta=1$ and any $B_{2}$ can be transformed to the case with a fixed $B_{2}(=1)$ by considering $\bar{B}_{3}=B_{3} / B_{2}$, $\bar{a}_{3}=a_{3}+(1 / \pi) \ln B_{2}$, and $\bar{a}_{2}=a_{2}+(1 / \pi) \ln B_{2}$. Here the barred quantities refer to the case with $B_{2}=1$. Equations (3), (4), and (19) exhibit this remarkable scaling property. Suppose that for a given $\lambda=\lambda_{0}$ and $\beta=\beta_{0}(=1)$ we have the two-particle scattering length $a_{2}$ and a two-particle bound state of binding energy $B_{2}$. It is easy to see from Eq. (19) by scaling arguments that then one has a bound state of binding energy $B_{2}=1$ for $\beta=\beta_{0} / B_{2}^{1 / 2}$ and $\lambda=\lambda_{0} / B_{2}^{2 m}$ and from Eqs. (9) and (11) that $\bar{a}_{2}$ is the corresponding scattering length. A similar scaling argument applied to Eq. (15) reveals that under this modification the three-particle binding energy and scattering length get changed from $B_{3}$ and $a_{3}$ to $\bar{B}_{3}$ and $\bar{a}_{3}$, respectively.

In Ref. [4] it is demonstrated that the plot of $\bar{B}_{3}$ (= $\left.B_{3} / B_{2}\right)$ vs $\bar{a}_{2}$ is independent of the potential form factors. The plots for $m=1,2,4$, and 10 yielded essentially the same result. This demonstrated the model independence of the three-boson bound states in two dimensions. We have reconfirmed this model independence for other values of $m$.

Here we shall mainly concentrate on the three-boson scattering observables. In Fig. 1 we plot the scattering length $\bar{a}_{3}$ vs $\bar{a}_{2}$ for $m=1,2,4$, and 10 in Eq. (2). For small $\bar{a}_{2}$ this plot is completely universal with all $m$ yielding essentially the same result. For large $\bar{a}_{2}$ and $\bar{a}_{3}$ this model independence tends to break down as we start moving out of the weak-binding limit $\left(B_{2}^{1 / 2} / \beta \ll 1\right)$. In the actual calculation for the extreme point given by $100 \bar{a}_{2}=15$, $B_{2}^{1 / 2} / \beta \approx 0.35(m=1), 0.24(m=2), 0.01(m=4)$, and $0.08(m=10)$. We see that the smaller values of $m$ have deviated more from the weak-binding limit compared to the larger values of $m$. This justifies the deviations of the $m=1$ and 2 curves from the $m=4$ and 10 curves for large $\bar{a}_{2}$. The $m=10$ curve in this

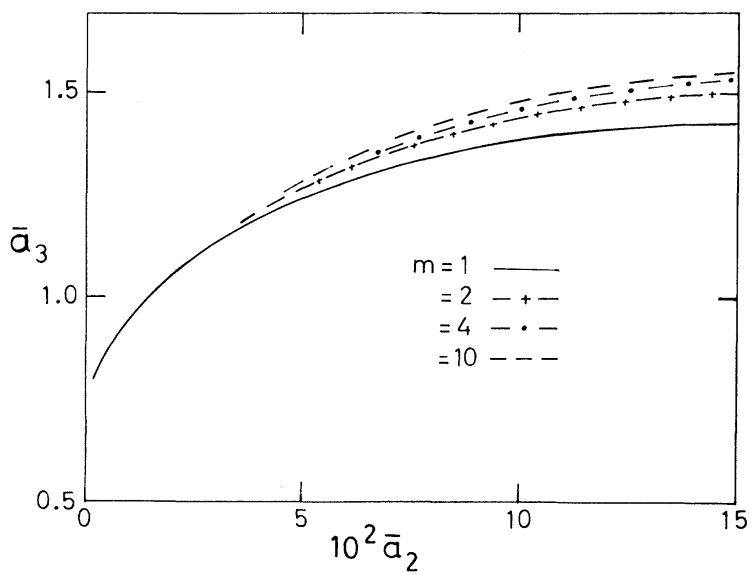

FIG. 1. The three-boson scattering length $\bar{a}_{3}$ plotted vs the two-boson scattering length $\bar{a}_{2}$ for the separable potential (2) with $m=1,2,4$, and 10. case is closer to the universal weak-binding limit. Even in the extreme point $100 \bar{a}_{2}=15$ the dipersion in $\bar{a}_{3}$ is small and is of the order of $10 \%$. This case is comparable to the three-dimensional three-nucleon system where the range parameter $\beta=1.4 \mathrm{fm}$ and the square root of the deuteron binding is $B_{2}^{1 / 2}=0.23 \mathrm{fm}$ [17]. However, in the three-nucleon system the dispersion in the value of the $S$-wave neutron-deuteron scattering length $a_{3}$ is much larger, with $a_{3}$ varying from $-1 \mathrm{fm}$ to $1 \mathrm{fm}$. Hence, unlike in the three-dimensional case, in the weakbinding limit $\bar{a}_{2}$ and $B_{2}(=1)$ are enough to determine the scattering length $a_{3}$ of one boson on the bound state of two bosons independent of the shape of the interaction potential as is obvious from Fig. 1.

In Fig. 2 we plot $\bar{a}_{3}$ vs $\bar{B}_{3}$ for $m=1$ and 10 . The plots for $m=2$ and 4 lie between those for $m=1$ and 10. For small $\bar{a}_{3}$ the model independence of the result is obvious. As in Fig. 1 this model independence starts to break down as $\bar{a}_{3}$ increases and we move out of the region of weakly bound two-particle bound states. This plot should be compared to the Phillips [19] plot of the threenucleon system, which yields a linear correlation between the triton-binding energy and the $S$-wave spin-doublet neutron-deuteron scattering length while different potential models with fixed two-nucleon binding and scattering lengths are employed. However, there is an interesting difference between the Phillips plot and the present plot. The present variation of $\bar{B}_{3}$ and $\bar{a}_{3}$ arises due to the variation of $\bar{a}_{2}$ while in the correlation of the Phillips plot both two-nucleon binding and scattering length are fixed. From Figs. 1 and 2 we realize that once we hold both the two-particle scattering length $a_{2}$ and binding energy $B_{2}$ fixed this will correspond to a unique scattering length $a_{3}$ and binding energy $B_{3}$ with an estimated dispersion of $10 \%$. In other words, in two dimensions with fixed $a_{2}$ and $B_{2}$, the $\bar{a}_{3}$ vs $\bar{B}_{3}$ plot reduces practically to a point with some dispersion, in contrast to the three-dimensional three-nucleon system. Consequently, the weakly bound three-dimensional three-nucleon system is model dependent or sensitive to the off-shell behavior of the two-nucleon interaction potential, whereas

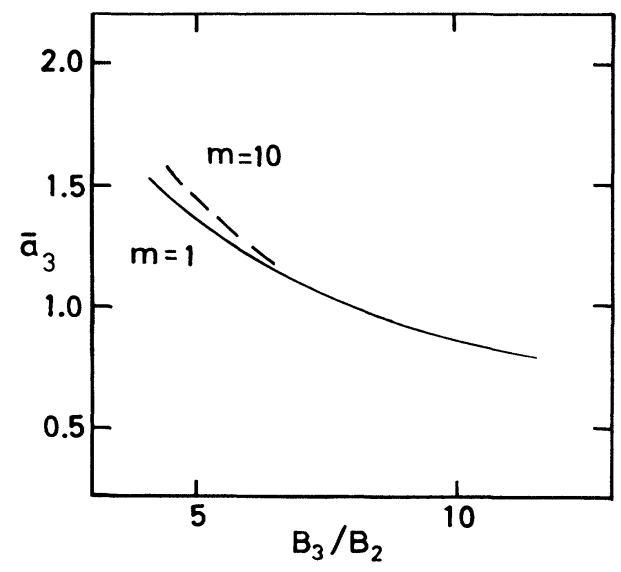

FIG. 2. The three-boson scattering length $\bar{a}_{3}$ plotted vs the three-boson binding $\bar{B}_{3}\left(=B_{3} / B_{2}\right)$ for the separable potential (2) with $m=1$ and 10 . 


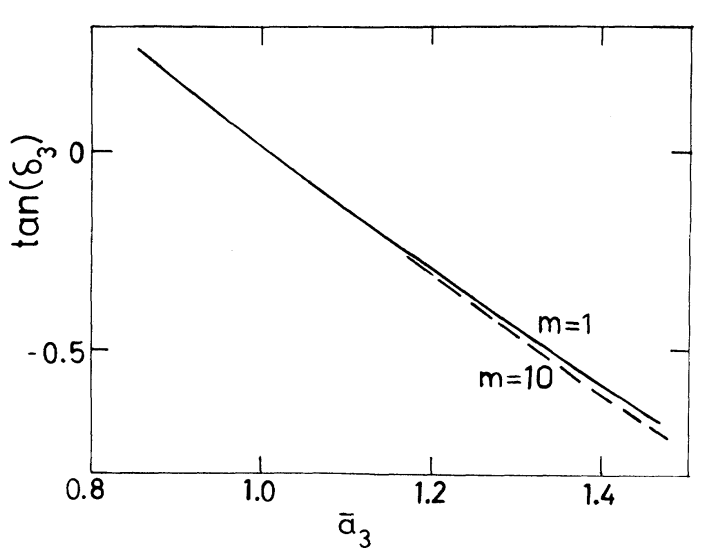

FIG. 3. The tangent of the three-boson phase shift $\tan \delta_{3}$ of Eq. (31) plotted vs the three-boson scattering length $\bar{a}_{3}$ for the separable potential (2) with $m=1$ and 10 .

the two-dimensional three-boson system in identical situations is virtually model independent and insensitive to the off-shell behavior of a two-particle interaction.

Finally, we see that this model independence of the three-boson binding energy and scattering length in two dimensions can be extended to the scattering phase shifts $\delta_{3}$ below the breakup threshold of a boson on a two-boson bound state. In order to test this we calculated $\delta_{3}$ for $k=1$ and $B_{2}=1$. This corresponds to a three-particle center-of-mass energy $s$ given by Eq. (14). In this case we plot $\tan \delta_{3}$ vs $\bar{a}_{3}$ in Fig. 3. As in Figs. 1 and $2 \bar{a}_{2}$ is varying in this calculation. For small $\bar{a}_{3}$ again the result is model independent. When $\bar{a}_{3}$ and $\bar{a}_{2}$ increases this model independence starts to break down as in Figs. 1 and 2. This plot should be compared to that of Frederico and Goldman [20] (FG) for the three-dimensional three-nucleon system, where they plotted $k \cot \delta$ ( $\delta$ being the nucleon-deuteron $S$-wave scattering phase shift) versus the nucleon-deuteron scattering length and obtained a linear correlation as in the Phillips plot. This study of FG is the three-dimensional analog of the present study in two dimensions in Fig. 3. However, in the study of FG both the two-nucleon binding energy and scattering length are held fixed. In the three-nucleon system even in the weak-binding limit, in contrast to the two-dimensional three-boson case, the result is model dependent. In the present study the two-particle binding energy is fixed but the two-particle scattering length is varying. Once the corresponding quantities $\left(B_{2}\right.$ and $\left.a_{2}\right)$ are held fixed in the present study we have only a sec- tion of the present plot in Fig. 3, which is just a point with small dispersion. Again we reach the same conclusion of model independence (off-shell independence) of the three-particle scattering observables in two dimensions.

\section{SUMMARY}

In this paper we have presented a systematic study of scattering and bound states of three identical bosons in two space dimensions at low energies using the Faddeev equations in the momentum space. The two-particle potential is taken to be of the $S$-wave separable type with varying form factors. In this case the Faddeev equations reduce to one-variable integral equations, which we refer to as the Amado-Lovelace-Mitra equations. One of the purposes of the present study is to investigate the model (in)dependence of the results once the two-particle binding energies and scattering lengths are held fixed. In the limit of weak two-particle binding $\left(B_{2}^{1 / 2} \ll \beta\right)$ we find the result of both scattering and bound states of the threeboson system to be independent of the variation of the potential shape provided that the two-particle scattering length and binding energy are held fixed. In the threedimensional three-particle system the results for threeparticle bound states and scattering are sensitive to the shape of the potential employed, even when the twoparticle binding energy and scattering lengths are held fixed. This behavior of the three-particle system in two dimensions has been justified by considering the analytic behavior of the dynamical equation (15) and has been related to the absence of the Thomas effect in this case [4]. The present finding is expected to have interesting consequences in the study of several few-particle systems in two dimensions. We have made a limited model study but we have argued that our conclusions are expected to be generally valid provided that we are limited to low energies and consider only the case of weak two-particle binding: $B_{2}^{1 / 2} \ll \beta$, where $B_{2}$ is the two-particle binding and $\beta$ is the inverse range parameter of the two-particle interaction.

\section{ACKNOWLEDGMENTS}

We thank Professor Akira Suzuki for interesting discussions. AD thanks Professor E.F. Redish for the hospitality at the University of Maryland. The work is supported in part by the Conselho Nacional de Desenvolvimento - Científico e Tecnológico (CNPq) of Brazil.
[1] M. L. Cramer, L. W. Bruch, and F. Cabral, J. Chem. Phys. 67, 1442 (1977); L. W. Bruch and J. A. Tjon, Phys. Rev. A 19, 425 (1979); F. Cabral and L. W. Bruch, J. Chem. Phys. 70, 4669 (1979).

[2] J. A. Tjon, Phys. Rev. A 21, 1334 (1980).

[3] L. P. H. de Goey, J. P. J. Driessen, B. J. Verharr, and J. T. M. Walraven, Phys. Rev. Lett. 53, 1919 (1984).
[4] S. K. Adhikari, A. Delfino, T. Frederico, I. D. Goldman, and L. Tomio, Phys. Rev. A 37, 3666 (1988).

[5] C. T. Christou and M. I. Haftel, Few-Body Systems 9, 195 (1990); 7, 1 (1989); M. I. Haftel and T. K. Lim, J. Chem. Phys. 84, 4407 (1986).

[6] L. P. H. de Goey, T. H. M. van der Berg, N. Mulders, H. T. C. Stoof, B. J. Verhaar, and W. Glöckle, Phys. Rev. 
B 34, 6183 (1986); L. P. H. de Goey et al., ibid. 38, 646 (1988); ibid. 38, 1150 (1988).

[7] H. F. Hess et al., Phys. Rev. Lett. 51, 483 (1983); 52, 1520 (1984).

[8] R. Sprik, J. T. M. Walraven, and I. F. Silvera, Phys. Rev. Lett. 51, 479 (1983); Phys. Rev. B 32, 5668 (1985).

[9] A. Lagendijk, Phys. Rev. B 25, 2054 (1982); E. V. L. Mello, J. J. Rehr, and O. E. Vilches, ibid. 28, 3859 (1983).

[10] J. G. Dash, Phys. Rep. 38C, 177 (1978).

[11] M. V. N. Murthy, J. Law, M. Brack, and R. K. Bhaduri, Phys. Rev. Lett. 67, 1817 (1991); M. Sporre, J. J. M. Verbaarschot, and I. Zahed, ibid. 67, 1813, (1991); A. Suzuki, M. K. Srivastava, R. K. Bhaduri, and J. Law, Phys. Rev. B 44, 10731 (1991); R. K. Bhaduri, R. S. Bhalerao, A. Khare, J. Law, and M. V. N. Murthy, Phys. Rev. Lett. 66, 523 (1991); A. Comtet, J. McCabe, and S. Ouvry, Phys. Lett. 260B, 372 (1991).

[12] R. L. Siddon and M. Schick, Phys. Rev. A 9, 907 (1974); S. K. Adhikari and R. D. Amado, Phys. Rev. Lett. 27, 485 (1971); W. G. Gibson, Mol. Phys. 49, 103 (1983);
Phys. Rev. A 36, 564 (1987).

[13] T. Frederico and I. D. Goldman (unpublished).

[14] L. H. Thomas, Phys. Rev. 47, 903 (1935); V. Efimov, Phys. Lett. 33B, 563 (1970).

[15] L. R. Dodd, J. Math. Phys. 11, 207 (1970).

[16] S. K. Adhikari, Am. J. Phys. 54, 362 (1986); S. K. Adhikari, W. G. Gibson, and T. K. Lim, J. Chem. Phys. 85, 5580 (1986).

[17] S. K. Adhikari and K. L. Kowalski, Dynamical Collision Theory and Its Applications (Academic, Boston, 1991).

[18] L. D. Faddeev, Mathematical Aspects of the Three-Body Problem in Quantum Scattering Theory (Davey, New York, 1965); R. D. Amado, in Elementary Particle Physics and Scattering Theory, edited by M. Chretien and S. S. Schweber (Gordon and Breach, New York, 1970), Vol. 2, p. 1; C. Lovelace, Phys. Rev. 135, B1225 (1964); A. N. Mitra, Adv. Nucl. Phys. 3, 1 (1969).

[19] A. C. Phillips, Rep. Prog. Phys. 40, 905 (1977).

[20] T. Frederico and I. D. Goldman, Phys. Rev. C 36, 1661 (1987). 